\title{
Bronchiectasis
}

This factsheet explains what bronchiectasis is, what causes it, and how it is diagnosed and managed. More detailed information is available on the Bronchiectasis Patient Priorities website: www.europeanlunginfo.org/bronchiectasis

\section{What is bronchiectasis?}

Bronchiectasis is a long-term lung condition. If you have bronchiectasis, your airways (tubes connecting your windpipe to the lowest part of the lungs) are wider than normal. This leads to mucus (or sputum) building up and making you more at risk of getting lung infections.

Infections can cause the lungs to become inflamed, which can damage or block parts of the lung, leading to symptoms like breathlessness, chest pain and tiredness.

Bronchiectasis is also known as non-cystic

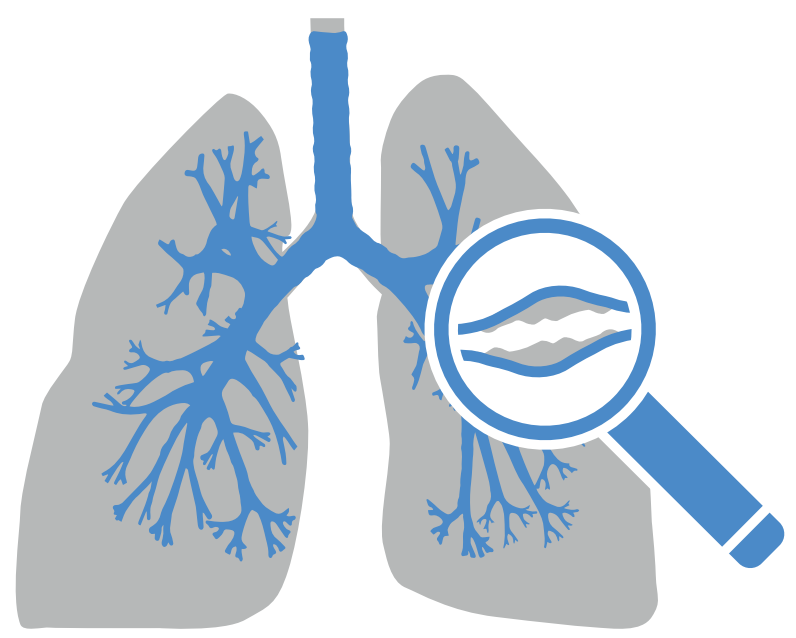
fibrosis (non-CF) bronchiectasis.

\section{What causes bronchiectasis?}

Many different things can cause bronchiectasis. In some cases it can be caused by a bad infection, such as pneumonia or childhood whooping cough. This is called post-infective bronchiectasis.

Other conditions that are often linked to or can cause bronchiectasis are:

- Severe asthma complicated by an allergic reaction to a fungus called Aspergillus - this is called allergic bronchopulmonary aspergillosis (ABPA)

- Conditions where the immune system attacks the body, like rheumatoid arthritis or ulcerative colitis 
- An obstruction or blockage of the airways after inhaling something

- Primary ciliary dyskinesia (PCD) - a genetic, long-term condition some people are born with where the lungs do not develop normally

- Chronic obstructive pulmonary disease (COPD) - a long-term condition that causes inflammation in the lungs, damaged lung tissue and a narrowing of the airways, making breathing difficult

- Alpha-1 antitrypsin deficiency - a genetic condition that can cause lung and liver problems

- Tuberculosis (TB) or non-tuberculous mycobacterial (NTM) infections - infections that often affect the lungs caused by the group of bacteria called mycobacteria Your doctor will ask you some questions and may do some tests to find out why you have bronchiectasis. Often the cause is not found (and is then called idiopathic bronchiectasis). This does not mean that there is no cause, just that it is not known.

The basic treatment of bronchiectasis is usually the same, no matter the cause. However, some causes call for a particular type of additional treatment.

\section{How is bronchiectasis diagnosed?}

The common symptoms of bronchiectasis are having a cough that is hard to get rid of, coughing up mucus, being short of breath and getting lots of lung infections. These symptoms are seen in other more common lung conditions like COPD or asthma, so most people are tested for them first. However, it is possible to have two, or even all three, of these conditions at the same time.

If your healthcare professional thinks you have bronchiectasis, they will do some extra tests.

\section{Common first tests}

- Chest X-ray: bronchiectasis cannot usually be seen in an X-ray, but it can help rule out other conditions.

- Spirometry: this is a breathing test that involves blowing hard and fast into a machine, emptying the lungs of air. It will not diagnose bronchiectasis, but is one way of measuring whether the lungs are damaged.

- Sputum samples: your doctor or nurse may ask you to cough some sputum into a pot for testing in a laboratory. This can identify bacteria in the lungs, in order to find the best antibiotic to use if you have a chest infection.

- Blood test: this can help to check your general health. 


\section{Specialist tests}

- CT (computed tomography) scan: this test is needed to diagnose bronchiectasis. This is where your body is X-rayed at a number of angles before a computer puts together a detailed image.

- Tests of the immune system: your doctor might organise blood tests to check how your immune system works and to check for possible causes of bronchiectasis, such as a lack of antibodies that fight infection or severe allergies.

- Breathing tests: spirometry (see above) may be performed, along with other more detailed lung function tests.

\section{What are the symptoms of bronchiectasis?}

Each person's experience is different, so it is not possible to describe a typical person with bronchiectasis.

However, you will have some or all of the following symptoms:

- Cough - which often involves coughing up mucus (sputum). The amount of sputum coughed up can vary a lot, as can the colour (which may be white, yellow, green, dark green or brown).

- Breathlessness - caused by the damaged airways working less well. In many cases, breathlessness is not a problem, but sometimes people may notice they are out of breath when walking uphill or up stairs. In severe cases people may get breathless during less strenuous exercise.

- Feeling very tired - the body uses a lot of energy to fight infections, as well as to cough and breathe. This can be made worse if people have trouble sleeping due to other symptoms.

- Chest discomfort - this can be a pain, tightness or a feeling of 'fullness' in the chest.

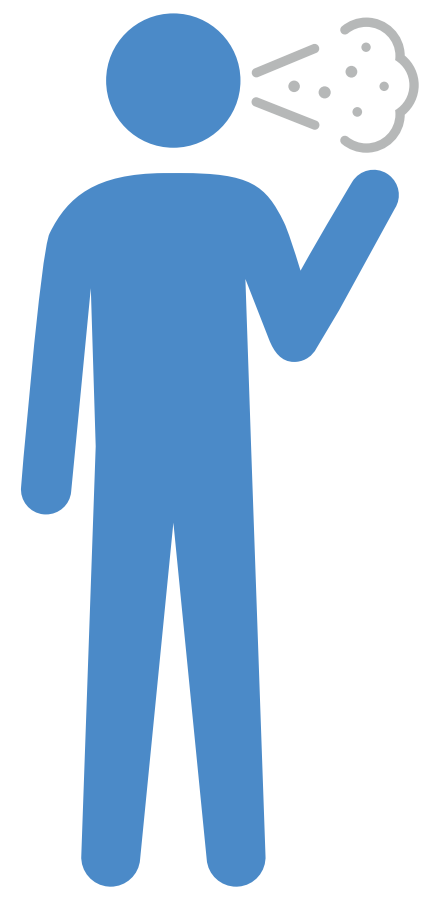

- Chest infections, also called exacerbations - you might easily catch infections that more quickly affect your chest. Other symptoms may get worse during an infection.

If properly treated and monitored, most people with bronchiectasis have a normal life expectancy. People with bronchiectasis are more likely to die because of other medical conditions that affect all people (like heart disease or cancer) than to die directly from bronchiectasis. 


\section{How severe is my condition?}

Bronchiectasis is a complicated condition and there is not one single test or sign of whether your condition is mild, moderate or severe. However, if your bronchiectasis is well controlled you should have few symptoms and be able to lead a normal life. Your bronchiectasis will be considered to be more severe when symptoms start to affect daily activities.

Factors linked with more severe bronchiectasis include:

- Breathlessness: being unable to walk 100 metres without stopping

- Chest infections: having three or more in a year

- Regularly growing bacteria on mucus (sputum) samples: this suggests your lungs have some regular infection, particularly if a bug called Pseudomonas is regularly found

- If a CT scan shows that both lungs, or several parts of the lung, are affected rather than a small part of the lung

- Hospital admissions: if you have had to go to hospital with bad chest infections or because you needed antibiotic injections

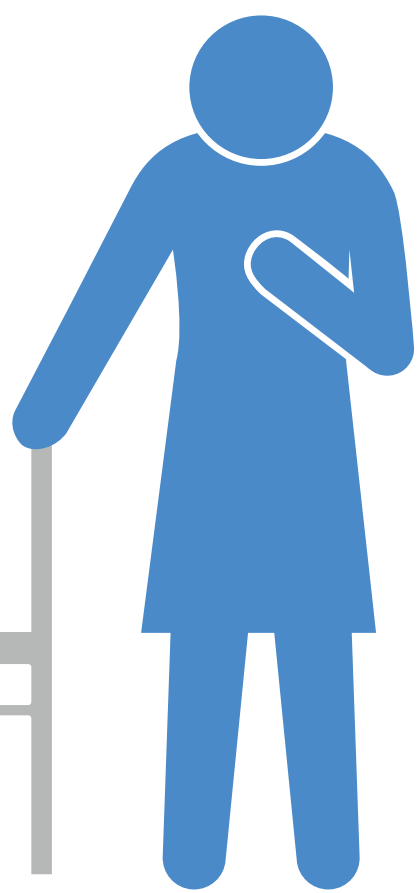

- Weight loss: if having bronchiectasis causes you to become underweight

- Lung function tests: having lower lung capacity

- Getting older: the lungs naturally get smaller and your immune system gets less effective as you age, which can impact on symptoms and severity of bronchiectasis

These are only a guide - some people may have several of these and still live a normal life; while some people may have none of the above, but have other important factors that cause them to feel more unwell. Many of these factors can improve with effective treatment.

People with severe bronchiectasis are at risk of a reduced life expectancy, which is why they should do everything they can to keep their lungs healthy. 


\section{How is bronchiectasis treated?}

Treatment for people with bronchiectasis has several aims:

- To prevent chest infections (exacerbations), during which symptoms get worse

- To treat symptoms

- To improve quality of life

- To stop the condition from getting worse

With good treatment, it is possible for people with bronchiectasis to remain stable for many years and to have good control of their symptoms.

The following are some of the commonly used treatments for bronchiectasis, including those recommended to doctors as part of the European bronchiectasis guidelines. Not all of these treatments are available or used in all European countries. If you have any questions regarding these treatments, please discuss them with your doctor.

\section{Treatment for inflammation}

- Inhalers: some inhalers contain medicine that opens up the airways to make it easier to breathe and reduce inflammation in the lungs.

- Tablets: some medications can also reduce inflammation. This includes a type of antibiotic called macrolides, which can reduce inflammation as well as treat infection.

\section{Treatment for infection}

- Vaccines: having an annual flu vaccine and a pneumococcal vaccine (which protects against the most common bacterial cause of pneumonia) is important to lower the risk of getting these.

- Antibiotics: any chest infections should be treated quickly with antibiotics - taken as tablets, through a mask (nebuliser) or as injections.

- Preventative antibiotics (in some cases): if you get lots of chest infections or have very severe symptoms, you may be given long-term antibiotic treatment to suppress the bacteria in the lungs.

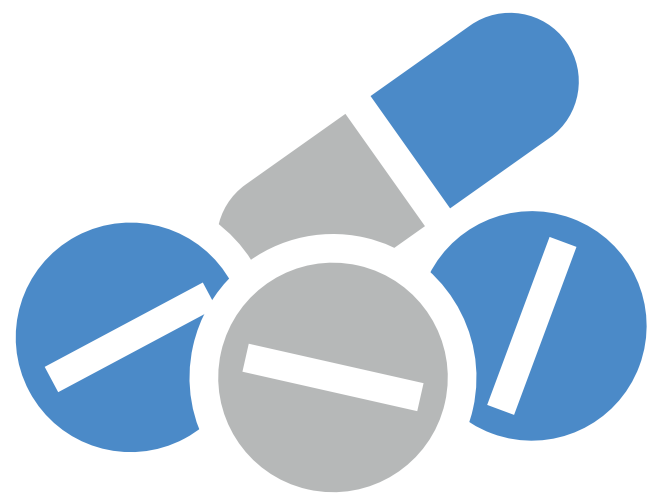


The doses are lower than those given to treat an infection, and are often given for at least 1 year and sometimes longer. They will not work straight away and need to be taken regularly to have their full effect. These medications can interact with other tablets, so it is important to tell other doctors that you are taking regular antibiotics before they prescribe you other drugs.

- Clearance of bacteria like Pseudomonas: Pseudomonas aeruginosa, or Pseudomonas, is a bug that can cause infection in people with bronchiectasis. If you catch Pseudomonas your doctor may recommend a course of antibiotics to try to get rid of or control it, or that you take a different antibiotic when you get chest infections in the future. People with Pseudomonas can sometimes have more chest infections and complications because of its resistance to antibiotics, and sometimes it can damage the lung. Guidelines for doctors recommend that people with Pseudomonas are monitored more regularly.

\section{Treatment for lung damage}

- Physiotherapy and airway clearance exercises can help to clear mucus (visit the Bronchiectasis Patient Priorities website for advice and video demonstrations)

- Medications can help to clear mucus (mucoactive drugs). These should be combined with regular physiotherapy exercises to be most effective.

- Bronchodilator inhalers relax the muscles in the airway.

- Regular exercise helps to clear sputum and encourages the lungs to work better.

Other important considerations are to avoid smoking and to treat the underlying cause of bronchiectasis if this has been identified.

\section{Other types of treatment}

\section{Oxygen therapy}

If your oxygen levels are low, you may need to breathe oxygen in through a tube or a mask. This could be short term (e.g. when you are in hospital with a chest infection until your levels return to normal) or long term if your lung has been damaged and is no longer able to take in enough oxygen.

You may also need additional oxygen during flights. Speak to your healthcare professional about this before you travel. 


\section{Surgery}

In most cases bronchiectasis can be well controlled and cared for without surgery. Surgery can sometimes be helpful if bronchiectasis affects only a small part of the lung and so might be cured by removing it, or if a section of the lung has been very badly damaged and is causing a lot of infections.

\section{Pulmonary rehabilitation}

You may be offered pulmonary rehabilitation as a way of improving your physical strength and reducing the impact of symptoms on your life.

Pulmonary rehabilitation is a type of treatment that aims to reduce the physical and emotional impacts of a lung condition on a person's life. It is a personalised programme that combines exercise training with education about ways you can help keep yourself as healthy as possible.

\section{Support to stop smoking}

Smoking does not cause bronchiectasis, but it can make your condition and symptoms much worse.

There is help available for you to stop smoking and alternatives to make quitting easier. Talk to your healthcare professional about how they can support you.

\section{Self-management}

Self-management is very important for people with long-term conditions like bronchiectasis. There is a range of different things you can do day-to-day to manage your condition. These include taking your medications and doing airway clearance exercises as recommended; monitoring your symptoms and telling your healthcare professional about any changes; being physically active; eating healthy foods; and, if you smoke, stopping smoking.

You can get more tips on self-management on the Bronchiectasis Patient Priorities website.

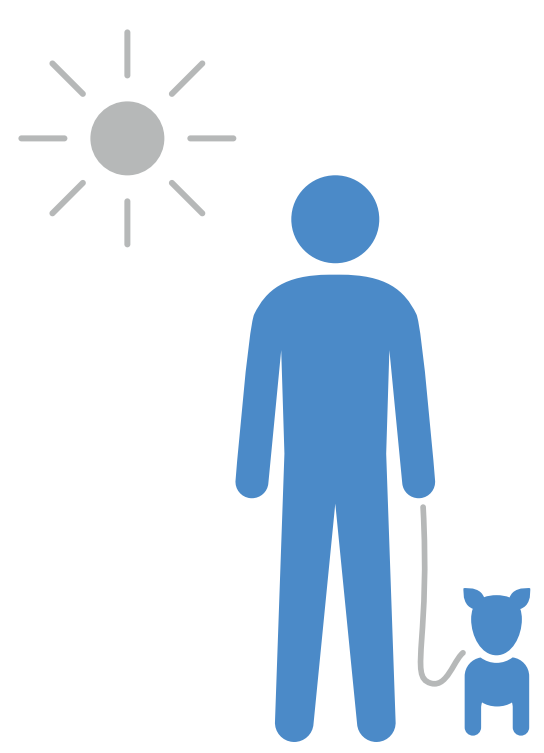

\section{Specialist care}

Treatment of bronchiectasis is best provided by a team of healthcare professionals that are experienced with the condition. In many countries there are now specialised clinics dedicated to people with bronchiectasis. 
These clinics will all be slightly different but will include:

- A doctor, or team of doctors, with a specialist interest and experience in bronchiectasis

- A physiotherapist, or team of physiotherapists, that can teach chest clearance exercises

- Nurses or other healthcare professionals that can provide education and support

- Services to provide inhaled/nebulised antibiotics, other specialised medications and intravenous antibiotics at home

- Specialised tests of the immune system or body to diagnose the cause of bronchiectasis

Some people with bronchiectasis regularly attend specialist clinics, while some might go once, or on a few occasions, and then be transferred back to the care of their regular doctor.

If you think you need to see a specialist, please discuss this with your doctor. There is no directory of bronchiectasis specialists across Europe, but if you have difficulty finding one, please contact the European Lung Foundation.

\section{Further reading}

\section{Bronchiectasis Patient Priorities | www.europeanlunginfo.org/bronchiectasis}

Information and support for people diagnosed with bronchiectasis and their caregivers, developed with the help of patients and healthcare professionals across Europe. A more detailed version of this information and a lay version of the European Respiratory Society guidelines on managing bronchiectasis are available on the website.

\section{European Lung Foundation | www.europeanlung.org}

The European Lung Foundation has a range of factsheets on topics relevant to bronchiectasis and its related conditions on its website.

\section{EMBARC | www.bronchiectasis.eu}

EMBARC is a pan-European network committed to promoting clinical research and education in bronchiectasis, through sharing of protocols, research ideas and expertise.

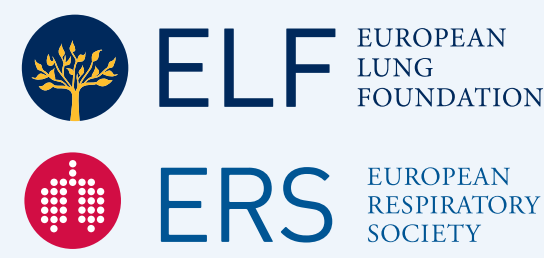
influence respiratory medicine. ELF is dedicated to lung health throughout Europe, and draws together the leading European medical experts to provide patient information and raise public awareness about lung disease. 